\title{
Contextual Interaction Design Research: Enabling HCI
}

\author{
Martin Murer, Alexander Meschtscherjakov, Verena Fuchsberger, Manuel Giuliani, \\ Katja Neureiter, Christiane Moser, Ilhan Aslan, Manfred Tscheligi \\ Center for Human-Computer Interaction \\ Department of Computer Sciences, University of Salzburg \\ Sigmund Haffner Gasse 18, 5020 Salzburg, Austria \\ \{firstname. lastname\}@sbg.ac.at
}

\begin{abstract}
Human-Computer Interaction (HCI) has always been about humans, their needs and desires. Contemporary HCI thinking investigates interactions in everyday life and puts an emphasis on the emotional and experiential qualities of interactions. At the Center for Human-Computer Interaction we seek to bridge meandering strands in the field by following a guiding metaphor that shifts focus to what has always been the core quality of our research field: Enabling HCI, as a leitmotif, draws our attention to how each research activity may benefit desires, goals and objectives. Enabling expresses how humancomputer interactions are always situated within someone's life, values, and needs; it emphasizes the power of thoughtful design to enable desired interactions and explore desirable futures and expresses how our research addresses the very essence of contextual qualities. This is facilitated through context-rich lab spaces that foster a maker culture for exploring novel forms of contextual interaction. Being an interdisciplinary research group that is rooted in the rich epistemological tradition of the field allows us to bridge boundaries between contemporary thinking and formerly prevalent domains, between established methodology and current research questions. In this paper we highlight how our organizational structure fosters this viewpoint on human-computer interactions.
\end{abstract}

\section{Contextual.Interaction.Design.Research}

In our research activities (e.g., a seven-year basic research project on „Contextual Interfaces", focusing on contextual qualities and interaction in factories and cars) we acknowledge that interactions are heavily dependent on the concrete, situated characteristics of the context in which they take place. This understanding results in a methodological viewpoint, which we entitle 'contextual.interaction.design.research'. Contextual research expresses our devotion to rich ethnomethodologically informed studies as well as the development and refinement of according methods. Our interaction designs are on one hand informed by those studies, aiming for user experiences that reflect the situated qualities of the context we address. On the other hand, we consider design methods and processes as legitimate mode of inquiry, as means of critical reflection and exploration. With interaction design research we investigate and discuss the according methodological challenges and opportunities. 


\section{Enabling HCI - The Case of six Research Groups}

The Center is organized in six research groups that address particular aspects of $\mathrm{HCI}$ in its relation to specific areas of human life. While the respective epistemologies differ, Enabling HCI guides all of them. With Contextual Persuasion we seek to enable individuals and groups to change their attitude and behavior by means of interactive technologies. Research includes fieldwork in persuasive potentials, design of behavior change systems, and in-situ studies of persuasive technologies. With research on Human-Robot Interaction we study how multimodal and natural interaction can enable social interaction with robots in challenging contexts such as industrial production, tele-operated medicine, and robots in public spaces.

We aim to enable new qualities of interaction through enhancing the knowledge about experiential and material use and design practices. This includes theoretical framings of the materiality of interactions, user experience, and constructive design research (Material \& Experiential Foundations). By Embodied Interaction Design we explore sensorimotor skills and couplings to enable extended bodily perception and experiences. Through reflective design, engineering, and craft we seek to understand the material qualities of novel technologies.

The group on Cooperation Experience aims to enable and understand meaningful computer mediated cooperation by investigating needs for successful cooperative processes (e.g., social presence) as well as identifying the nature of resources within social cooperation (social capital). Enhancing Life Experiences seeks to enable and promote social interaction and health/well-being of selected user groups in the (extended) home by creating valuable technical solutions in a user-centered design approach. The respective focus lies on enhancing peoples' life experiences.

The different research topics are bridged with at the moment two dedicated contextual laboratories (Car Interaction Lab and Fabrication Experience Lab) that allow our researchers to design, prototype, and study contextual interactions.

\section{Conclusion}

The variety of viewpoints has allowed us to design new experiences through different HCI lenses with a particular focus on the context in which the interaction takes place, emphasizing the essence of interaction through making and reflection processes in our research. It enables us to focus our research on different core topics in HCI and at the same time use dedicated contexts as enabling design and research spaces. By building an environment in which researchers from different HCI domains can collaboratively create meaningful and tangible interaction designs in an atmosphere that is charged with experiential qualities of the contexts we address, we enrich both the design process, as well as the outcome of our research efforts. With contextual.interaction.design.research we have been able to identify relevant aspects of the constant, situated interplay between users and artifacts in order to create artifacts that enable humans to experience novel, exceptional, or disruptive interactions, aiming to go beyond current trends and imaginations. 